\title{
Platelet-to-Lymphocyte ratio (PLR) is a Novel Independent Risk Factor for Newborn Patients in the Neonatal Intensive Care Unit (NICU)
}

\author{
Yanfei Tang \\ Jiaxing University \\ Yiqun Teng \\ Jiaxing University \\ Lingyan Xu \\ Jiaxing University
}

Guangtao Xu ( $\nabla$ gtxu@zjxu.edu.cn )

Jiaxing University https://orcid.org/0000-0001-5846-9280

\section{Deqing Chen}

Jiaxing University

Xin Jin

Jiaxing University

Wanlu Li

Jiaxing University

Xiuhui Jin

University of Toronto

Wen Zhu

Jiaxing University

Bo Hu

Jiaxing University

Ruilin Shen

Jiaxing University

Yuzhang Zhu

Jiaxing University

\section{Research}

Keywords: platelet-to-lymphocyte ratio, neonatal intensive care unit, risk factor, newborn, hospital mortality, length of ICU stay, length of hospital stay, 90-day mortality

Posted Date: June 28th, 2021 
DOl: https://doi.org/10.21203/rs.3.rs-624694/v1

License: (c) (1) This work is licensed under a Creative Commons Attribution 4.0 International License. Read Full License 


\section{Abstract}

Objective: To investigate the prognostic significance of the platelet-to-lymphocyte ratio (PLR) for newborn patients in the neonatal intensive care unit (NICU).

Design: A retrospective cohort study.

Setting and participants: Data on 5240 newborn patients in the NICU extracted from the Multiparameter Intelligent Monitoring in Intensive Care III (MIMIC III) database.

Methods: Spearman correlation was used to analyze the association of PLR with length of hospital and ICU stays. The chi-square test was used to analyze the association of PLR with mortality rate.

Multivariable logistic regression was used to determine whether the PLR was an independent prognostic factor of mortality. The area under the receiver operating characteristic (ROC) curve was used to assess the predictive ability of models combining PLR with other variables.

Results: PLR was negatively associated with length of hospital stay and ICU stay (hospital stay: Spearman's rho $=-0.416, P<0.0001$; ICU stay: Spearman's rho $=-0.442, P<0.0001)$. PLR was significantly correlated with hospital mortality $(P<0.0001)$. Lower PLR was associated with higher hospital mortality $(\mathrm{OR}=0.85,95 \% \mathrm{Cl}=0.75-0.95, P=0.005)$ and 90 -day mortality $(\mathrm{OR}=0.85,95 \% \mathrm{Cl}=0.76-0.96, P=0.010)$. The prognostic predictive ability of models combining PLR with other variables for hospital mortality was moderately good (AUC for Model 1=0.804; AUC for Model 2=0.964).

Conclusion: PLR is a novel independent risk factor for newborn patients in the NICU.

\section{Introduction}

The first month of life is riskiest time for child survival, accounting for approximately $40 \%$ of all childhood mortality ${ }^{1-3}$. Each year, 2.6 million neonates die globally, with $75 \%$ of neonatal deaths occurring in the first week of life and $99 \%$ of deaths occurring in low- and middle-income countries ${ }^{3,4}$. Premature delivery and birth-related complications (such as birth asphyxia and neonatal sepsis) are considered to be the main causes of neonatal death ${ }^{5}$.

In recent years, the neutrophil-to-lymphocyte ratio (NLR) ${ }^{6}$, lymphocyte-to-monocyte ratio (LMR) ${ }^{7}$ and platelet-to-lymphocyte ratio (PLR) ${ }^{8}$ have been found to be independent predictors of prognosis in various benign and malignant conditions ${ }^{9,10}$. Moreover, NLR, LMR and PLR were reported to be related to the outcome of intensive care unit (ICU) patients, because of their rapid response to systemic inflammation and stress ${ }^{11-13}$. However, little is known about the associations of NLR, LMR and PLR with prognosis in newborn patients in the neonatal intensive care unit (NICU).

The primary purpose of this study was to determine the associations of NLR, LMR and PLR with hospital mortality in newborn patients in the NICU. In the present study, we were the first to report that PLR can 
serve as an independent risk factor for newborn patients in the NICU.

\section{Methods}

\section{Data Source}

A retrospective cohort study design was used in this study. Data were obtained from the ICU database, a free accessible critical care database of Medical Information Mart for Intensive Care III (MIMIC-III). The clinical data of patients who stayed in the ICU of Beth Israel Deaconess Medical Center (BIDMC) between 2001 and 2012 were selected ${ }^{14}$. The institutional review boards of both the BIDMC and the Massachusetts Institute of Technology Affiliates approved the access to the database. No informed consent was required because all of the data were deidentified.

\section{Patient Selection}

Clinical data of eligible patients in the MIMIC-III database were selected for analysis in this study. The eligibility criteria were (1) newborn patents admitted to the NICU; and (2) patients with routine preoperative blood examinations within 24 hours of admission.

\section{Data Extraction}

All of the data were obtained and extracted by using the Structured Query Language (SQL), and pgAdmin4 for PostgreSQL was used as the administrative platform. The extracted data mainly included age, sex, birthweight, heart rate (HR), laboratory parameters (red blood cell count (RBC), peripheral white blood cell count (WBC), platelet count, lymphocyte count, neutrophil count, monocytes count), comorbidities (congestive heart failure, cardiac arrhythmias, valvular disease, pulmonary circulation disorder, hypertension, liver disease and renal failure), the Simplified Acute Physiology Score (SAPS) II, the Sequential Organ Failure Assessment (SOFA) score and the model for end-stage liver disease (MELD) score. PLR was calculated by dividing the platelet count by the lymphocyte count. NLR was calculated by dividing the neutrophil count by the lymphocyte count. LMR was calculated by dividing the lymphocyte count by the monocyte count. Given that the proportion of missing data for each variable was $<1.5 \%$, we directly omitted these data in further analyses.

\section{Outcome Variables}

The following outcome variables were extracted: hospital mortality, length of ICU stay, length of hospital stay and 90-day mortality (post-ICU admission). Because a patient may have had more than one ICU admission during a single hospitalization, the length of ICU stay was entirely determined by the first ICU hospitalization.

\section{Statistical Analysis}

Continuous variables are presented as the mean \pm standard deviation or the median (interquartile range), and were compared via $t$-test or the Mann-Whitney $U$ test. Categorical data are presented as numbers with 
proportions and were analyzed via the $\chi^{2}$ test. Correlation of length of ICU stay and hospital stay with the laboratory parameters were assessed with the nonparametric Spearman's rank correlation test. Logistic regression with the univariate and multivariate analyses was used to identify independent prognostic factors of mortality (hospital mortality and 90-day mortality) for newborn NICU patients. Two different models were designed to adjust for potential confounders. Model 1 was adjusted for NLR, LMR, MELD, SAPS II and liver disease. Moreover, Model 2 was adjusted for NLR, LMR and MELD. Receiver operating characteristic (ROC) curves were constructed, and the area under the curve (AUC), sensitivity and specificity were calculated. $P$-values of less than 0.05 were considered to indicate statistical significance.

\section{Results}

\section{Baseline Characteristics of the Study Population}

In total, 5240 patients who met the selection criteria were enrolled in our study, among whom 43 patients $(0.82 \%)$ died in the hospital. The baseline characteristics of the enrolled patients are summarized in the Table 1. 
Table 1

Baseline characteristics of the study population with different survival status in hospital

\begin{tabular}{|c|c|}
\hline Survivors & Nonsurvivors \\
\hline$(n=5197)$ & $(n=43)$ \\
\hline
\end{tabular}

\section{Demographics}

\begin{tabular}{llll} 
Age & $0.00139 \pm 0.00001$ & $0.00150 \pm 0.00011$ & 0.311 \\
\hline Male, n (\%) & $2823(53.87 \%)$ & $26(0.50 \%)$ & 0.420 \\
\hline Birthweight $(\mathrm{kg})$ & $1.63(1.10-2.48)$ & $0.84(0.62-1.59)$ & $<0.0001$ \\
\hline Heart rate $(\mathrm{bpm})$ & $152(140-169)$ & $150(140-168)$ & 0.001 \\
\hline Laboratory events & & & \\
\hline RBC, $10^{6} / \mathrm{L}$ & $4.14(3.70-4.62)$ & $3.80(3.45-4.23)$ & 0.001 \\
\hline WBC, $10^{9} / \mathrm{L}$ & $15.3(10.9-19.8)$ & $8.8(4.7-11.4)$ & $<0.0001$ \\
\hline Lymphocytes, \% & $29(21-42)$ & $54.55(33-67)$ & $<0.0001$ \\
\hline Neutrophils, \% & $57(42.9-66)$ & $27(19-46)$ & $<0.0001$ \\
\hline Monocytes, \% & $7(4-9.2)$ & $7(4-11)$ & 0.707 \\
\hline Platelets, $10^{9} / \mathrm{L}$ & $284(232-338)$ & $212(174-240)$ & $<0.0001$ \\
\hline LMR & $4.33(2.75-7.4)$ & $7.67(4.17-13)$ & $<0.0001$ \\
\hline NLR & $2(1.02-3.13)$ & $0.55(0.28-1.64)$ & 0.0008 \\
\hline PLR & $9.77(6.15-14.36)$ & $3.67(2.73-5.72)$ & $<0.0001$
\end{tabular}

\section{Comorbidities}

$\begin{array}{llll}\text { Congestive heart failure } & 14(0.27 \%) & 0(0.00 \%) & 0.736 \\ \text { Cardiac arrhythmias } & 19(0.36 \%) & 0(0.00 \%) & 0.694 \\ \text { Valvular disease } & 8(0.15 \%) & 0(0.00 \%) & 0.799 \\ \text { Pulmonary circulation disorder } & 5(0.10 \%) & 0(0.00 \%) & 0.840 \\ \text { Hypertension } & 10(0.19 \%) & 0(0.00 \%) & 0.773 \\ \text { Liver disease } & 2(0.03 \%) & 1(0.015 \%) & <0.0001 \\ \text { Renal failure } & 0(0.00 \%) & 1(0.015 \%) & <0.0001 \\ \text { Scores } & & & <0.0001 \\ \text { SAPS II } & 18.86 \pm 0.15 & 37.58 \pm 0.81 & \end{array}$




\begin{tabular}{|c|c|c|c|}
\hline & $\begin{array}{l}\text { Survivors } \\
(n=5197)\end{array}$ & $\begin{array}{l}\text { Nonsurvivors } \\
(n=43)\end{array}$ & $P$ value \\
\hline SOFA & $2.75 \pm 0.05$ & $10.91 \pm 0.41$ & $<0.0001$ \\
\hline MELD & $7.97 \pm 0.05$ & $11.64 \pm 0.70$ & $<0.0001$ \\
\hline \multicolumn{4}{|c|}{$\begin{array}{l}\text { Values are presented as the mean } \pm \text { standard deviation, median (interquartile range), or number of } \\
\text { patients (\%). }\end{array}$} \\
\hline \multicolumn{4}{|c|}{$\begin{array}{l}\text { WBC, white blood cell; SAPS II, Simplified Acute Physiology Score II; SOFA, Sequential Organ Failure } \\
\text { Assessment; MELD, model for end-stage liver disease }\end{array}$} \\
\hline
\end{tabular}

The demographic characteristics of the survivors and nonsurvivors are presented in Table 1. No significant differences were observed for age and sex between nonsurvivors and survivors. Nonsurvivors had a higher birthweight and HR. Moreover, nonsurvivors had much higher RBCs, WBCs, neutrophils, platelets, NLRs and PLRs. Nonsurvivors tended to have lower lymphocytes, LMRs, SAPS II scores, SOFA scores and MELD scores, as well as a history of liver disease and renal failure (Table 1).

\section{Association of Inflammatory Markers with Length of Hospital Stay and ICU Stay in Newborn Patients in the NICU}

LMR, NLR and PLR were reported to be related to the outcome of various diseases. To investigate the associations of these inflammatory markers with the length of hospital stay and ICU stay in newborn patients in the NICU, Spearman's rank correlation test was used, and the results are shown in Table 2. LMR was significantly positively associated with length of hospital stay and ICU stay (hospital stay: Spearman's rho $=0.228, P<0.0001$; ICU stay: Spearman's rho $=0.254, P<0.0001)$. Both NLR and PLR were negatively associated with length of hospital stay and ICU stay (for NLR, hospital stay: Spearman's rho $=-0.427, P<0.0001$; ICU stay: Spearman's rho $=-0.448, P<0.0001$. For PLR, hospital stay: Spearman's rho $=-0.416, P<0.0001$; ICU stay: Spearman's rho $=-0.442, P<0.0001)$.

Table 2

The correlation of LMR, NLR and PLR with hospital stay and ICU stay

\begin{tabular}{|lllll|}
\hline \multicolumn{3}{|l}{ Length of Hospital stay } & \multicolumn{2}{l|}{ Length of ICU stay } \\
\hline & Spearman's Rho & P value & Spearman's Rho & P value \\
\hline LMR & 0.228 & $<0.0001$ & 0.254 & $<0.0001$ \\
\hline NLR & -0.427 & $<0.0001$ & -0.448 & $<0.0001$ \\
\hline PLR & -0.416 & $<0.0001$ & -0.442 & $<0.0001$ \\
\hline
\end{tabular}

\section{Association of Inflammatory Markers with Hospital Mortality in Newborn Patients in the NICU}


In the present study, the correlation of the inflammatory markers with the mortality in the newborn patients in the NICU was investigated. Quartiles of LMR, NLR and PLR were significantly correlated with hospital mortality (all $P<0.0001$ ) (Table 3 ). A higher rate of hospital mortality was observed in patients in the fourth LMR quartile than is those in the first, second and third quartiles. For NLR and PLR, a higher rate of hospital mortality was observed in patients in the first quartile than in those in other quartiles.

Table 3

The relationship between LMR, NLR and PLR with Hospital mortality

\begin{tabular}{|c|c|c|c|c|c|}
\hline & Q1 & Q2 & Q3 & Q4 & $P$ value \\
\hline \multicolumn{6}{|l|}{ LMR } \\
\hline Survivors & $1364(26.03 \%)$ & $1288(24.58 \%)$ & $1288(24.58 \%)$ & $1257(23.99 \%)$ & \multirow[t]{2}{*}{$<0.0001$} \\
\hline Nonsurvivors & $3(0.06 \%)$ & $5(0.10 \%)$ & $6(0.11 \%)$ & $29(0.55 \%)$ & \\
\hline \multicolumn{6}{|l|}{ NLR } \\
\hline Survivors & $1285(24.52 \%)$ & $1277(24.37 \%)$ & $1284(24.50 \%)$ & $1351(25.78 \%)$ & \multirow[t]{2}{*}{$<0.0001$} \\
\hline Nonsurvivors & $31(0.59 \%)$ & $4(0.076 \%)$ & $4(0.076 \%)$ & $4(0.076 \%)$ & \\
\hline \multicolumn{6}{|l|}{ PLR } \\
\hline Survivors & $1258(24.01 \%)$ & $1283(24.48 \%)$ & $1288(24.58 \%)$ & $1368(26.11 \%)$ & \multirow[t]{2}{*}{$<0.0001$} \\
\hline Nonsurvivors & $32(0.61 \%)$ & $5(0.10 \%)$ & $2(0.038 \%)$ & $4(0.076 \%)$ & \\
\hline \multicolumn{6}{|c|}{ Q, Quartiles of LMR, NLR and PLR. } \\
\hline
\end{tabular}

Univariate logistic regression analysis was performed. As shown in Table 4, PLR, NLR and LMR were associated with hospital mortality and 90-day mortality (PLR for hospital mortality: OR $=0.76,95 \% \mathrm{Cl}=$ 0.69-0.83, $P<0.0001$. NLR for hospital mortality: $\mathrm{OR}=0.50,95 \% \mathrm{Cl}=0.37-0.69, P<0.0001$. LMR for hospital mortality: $\mathrm{OR}=1.03,95 \% \mathrm{Cl}=1.01-1.05, P<0.0001$. $\mathrm{PLR}$ for 90 -day mortality: $\mathrm{OR}=0.76,95 \% \mathrm{Cl}$ $=0.69-0.84, P<0.0001$. NLR for 90 -day mortality: $\mathrm{OR}=0.49,95 \% \mathrm{Cl}=0.36-0.68, P<0.0001$. LMR for $90-$ day mortality: $\mathrm{OR}=1.04,95 \% \mathrm{Cl}=1.02-1.05, P<0.0001)$. 
Table 4

Univariate Logistic regression analyses for prognosis in newborn patients

\begin{tabular}{|llll|}
\hline \multicolumn{1}{|l}{ Outcome } & OR & \multicolumn{1}{l}{$95 \% \mathrm{Cl}$} & P value \\
\hline \multicolumn{4}{|l}{ Hospital mortality } \\
\hline PLR & 0.76 & $0.69-0.83$ & $<0.0001$ \\
\hline NLR & 0.50 & $0.37-0.69$ & $<0.0001$ \\
\hline LMR & 1.03 & $1.01-1.05$ & $<0.0001$ \\
\hline MELD & 1.19 & $1.12-1.25$ & $<0.0001$ \\
\hline SAPS II & 1.17 & $1.13-1.21$ & $<0.0001$ \\
\hline Liver disease & 63.18 & $5.62-710.58$ & 0.001 \\
\hline 90-day mortality & & \\
\hline PLR & 0.76 & $0.69-0.84$ & $<0.0001$ \\
\hline NLR & 0.49 & $0.36-0.68$ & $<0.0001$ \\
\hline LMR & 1.04 & $1.02-1.05$ & $<0.0001$ \\
\hline MELD & 1.18 & $1.12-1.25$ & $<0.0001$ \\
\hline SAPS II & 1.17 & $1.13-1.22$ & $<0.0001$ \\
\hline Liver disease & 63.18 & $5.62-710.58$ & 0.001 \\
\hline
\end{tabular}

The results of the multivariate analysis are summarized in Table 5, and only PLR was significantly associated with hospital mortality (Model 1: $\mathrm{OR}=0.85,95 \% \mathrm{Cl}=0.75-0.95, P=0.005$; Model 2: $\mathrm{OR}=0.75$, $95 \% \mathrm{Cl}=0.67-0.84, P<0.0001$ ) and 90-day mortality (Model 1: $\mathrm{OR}=0.85,95 \% \mathrm{Cl}=0.76-0.96, P=0.010$; Model 2: $\mathrm{OR}=0.79,95 \% \mathrm{Cl}=0.71-0.89, P<0.0001)$ in all models. In the multivariate analysis, Model 1 was adjusted for NLR, LMR, MELD, SAPS II and Liver disease. Model 2 was adjusted for NLR, LMR and Meld. The data suggested that PLR could be an independent risk factor for hospital mortality and 90-day mortality in the newborn patients in the NICU. 
Table 5

Association between PLR with Prognosis of newborn patients

\begin{tabular}{|c|c|c|c|c|}
\hline & Model 1 & & Model 2 & \\
\hline Variable & OR $(95 \% \mathrm{Cl})$ & $P$ value & OR $(95 \% \mathrm{Cl})$ & $P$ value \\
\hline \multicolumn{5}{|c|}{ Hospital Mortality } \\
\hline PLR & $0.84(0.75-0.95)$ & 0.005 & $0.75(0.67-0.84)$ & $<0.0001$ \\
\hline NLR & $1.37(0.98-1.90)$ & 0.063 & $1.18(0.85-1.65)$ & 0.315 \\
\hline LMR & $1.01(0.99-1.03)$ & 0.253 & $1.01(0.99-1.03)$ & 0.377 \\
\hline MELD & $0.89(0.80-0.99)$ & 0.028 & $1.11(1.05-1.19)$ & $<0.0001$ \\
\hline SAPS ॥ & $1.19(1.14-1.26)$ & $<0.0001$ & & \\
\hline Liver disease & $11.39(0.84-154.04)$ & 0.067 & & \\
\hline \multicolumn{5}{|c|}{ 90-day Mortality } \\
\hline PLR & $0.85(0.76-0.96)$ & 0.010 & $0.79(0.71-0.89)$ & $<0.0001$ \\
\hline NLR & $1.32(0.94-1.86)$ & 0.106 & $1.15(0.82-1.63)$ & 0.417 \\
\hline LMR & $1.01(0.99-1.03)$ & 0.182 & $1.01(0.99-1.03)$ & 0.232 \\
\hline MELD & $0.88(0.79-0.98)$ & 0.017 & $1.12(1.04-1.19)$ & 0.001 \\
\hline SAPS II & $1.20(1.14-1.27)$ & $<0.0001$ & & \\
\hline Liver disease & $11.53(0.83-159.82)$ & 0.068 & & \\
\hline \multicolumn{5}{|c|}{ Model 1 was adjusted for NLR, LMR, Meld, SAPS II and Liver disease. } \\
\hline \multicolumn{5}{|c|}{ Model 2 was adjusted for NLR, LMR and MELD. } \\
\hline
\end{tabular}

Prognostic Predictive Ability of Models Combining PLR with Other Variables for Hospital Mortality in Newborn Patients in the NICU

To evaluate the predictive ability of models combining PLR and other clinical variables for hospital mortality, ROC curve analysis was performed, and the AUC for Model 1 (Model 1: PLR, LMR and NLR) was $0.804(95 \% \mathrm{Cl}=0.73-0.88, P<0.0001)$ and for Model 2 (Model 2: PLR, LMR, NLR, SOFA and MELD) was $0.964(95 \% \mathrm{Cl}=0.95-0.98, P<0.0001)$ (Fig. 1).

\section{Discussion}

In the present study, we found that LMR was significantly positively was associated with length of hospital stay and ICU stay while both NLR and PLR were negatively associated with length of hospital 
stay and ICU stay. PLR, NLR and LMR were associated with hospital mortality and 90-day mortality, but only PLR was significantly associated with hospital mortality and 90-day mortality in the multivariate analyses. The prognostic predictive ability of models combining PLR with other variables for hospital mortality was moderately good. To our knowledge, this is the first investigation to demonstrate that PLR can serve as an independent risky factor for newborn patients of NICU.

Inflammatory cells, including WBCs and their subtypes (such as lymphocytes, monocytes and neutrophils), have been well validated to play an indispensable role in various benign and malignant conditions. Moreover, platelets could play a critical role in the immunomodulatory and inflammatory process ${ }^{15}$, by inducing the release of inflammatory cytokines and interacting with different kinds of immune cells, such as neutrophils, T-lymphocytes, and macrophages (the precursors of macrophages are monocytes), which contribute to the initiation or exacerbation of the inflammatory process ${ }^{16,17}$. Low lymphocyte counts could represent a suppressed immune and inflammatory response, which is related to inflammatory disease ${ }^{18,19}$. Thus, PLR was proposed to serve as a novel systematic inflammatory indicator $^{20}$.

The association between PLR and outcomes was different in different cohorts. Both high and low PLR were associated with increased mortality, among critically ill patients with acute kidney injury $(A K I)^{21}$. In another study, high PLR was positively associated with increased epicardial adipose tissue deposition in diabetes patients ${ }^{22}$. Wang et al showed that high PLR was independently associated with shorter disease-free days and lower overall survival rates in lung adenosquamous carcinoma ${ }^{23}$. For fetal malnutrition, cord-blood PLR negatively correlated with term fetal malnutrition gestational age neonates ${ }^{24}$. Maternal PLR was negatively correlated with the week of birth and birth weight of the infant ${ }^{25}$. Our data showed that PLR was associated with the prognosis of newborn patients in the NICU. PLR was negatively associated with length of hospital stay and ICU stay. A higher rate of hospital mortality was observed in patients in the first PLR quartile than in those in the other quartiles. The prognostic predictive ability of models combining PLR with other variables for hospital mortality was moderately good (AUC for Model $1=0.804$; AUC for Model2 $=0.964$ ). These data suggested that PLR can serve as an independent risky factor for newborn patients in the NICU.

\section{Conclusion}

In summary, we demonstrated that lower PLR was significantly associated with higher hospital mortality. The prognostic predictive ability of models combining PLR with other variables for hospital mortality was moderately good. PLR is a novel independent risk factor for newborn patients in the NICU.

\section{List of Abbreviations}

AKI, acute kidney injury; AUC, area under the curve; BIDMC, Beth Israel Deaconess Medical Center, HR, heart rate; ICU, intensive care unit; LMR, lymphocyte-to-monocyte ratio; MIT, Massachusettes Institute of Technology; MELD, model for end-stage liver disease score; MIMIC III, Multiparameter Intelligent 
Monitoring in Intensive Care III database; NLR, neutrophil-to-lymphocyte ratio; NICU, neonatal intensive care unit; PLR, platelet-to-lymphocyte ratio; RBC, red blood cell; ROC, receiver operating characteristic curve; SOFA, Sequential Organ Failure Assessment score; SAPS II, Simplified Acute Physiology Score II; SQL, Structured Query Language; WBC, white blood cell.

\section{Declarations}

\section{Ethics approval and consent to participate}

The institutional review boards of the MIT (Cambridge, Massachusetts) and BIDMC (Boston, Massachusetts) reviewed and approved studies involving human participants. According to national laws and institutional requirements, this study does not require written informed consent.

\section{Consent for publication}

Not applicable.

\section{Availability of data and materials}

Authors will provide raw data to support the conclusions of this research without reservation.

\section{Competing interests}

The authors declare that the study was conducted in the absence of any commercial or financial relationship that could be constructed as a potential conflict of interest.

\section{Funding}

This work was financially supported by the Major Transverse Research Projects of Jiaxing University, China (00619006 to GX). The sponsors of the study were not involved in study design, data collection, data analysis, data interpretation, or writing of the manuscript.

\section{Authors' contributions}

YZ, YT and GX conceived and designed the study. GX and RS administratively supported this work. YT, YT, LX, DC, XJ, WL, XJ, WZ, and BH provided, selected, assembled, analyzed and interpretated data. All authors contributed toward data analysis, drafting and critically revising the paper, and agree to be accountable for all aspects of the work. All authors have read and confirmed that they meet ICMJE criteria for authorship.

\section{Acknowledgements}

We appreciate American Journal Experts (AJE) for their careful English language editing. We apologize to all those researchers whose work could not be cited due to space limitations. 


\section{References}

1. Orsido TT, Asseffa NA, Berheto TM. Predictors of Neonatal mortality in Neonatal intensive care unit at referral Hospital in Southern Ethiopia: a retrospective cohort study. BMC Pregnancy Childbirth. 2019;19:83.

2. Collaborators GBDCM. Global, regional, national, and selected subnational levels of stillbirths, neonatal, infant, and under-5 mortality, 1980-2015: a systematic analysis for the Global Burden of Disease Study 2015. Lancet. 2016;388:1725-74.

3. Lancet. 2014;384:957 - 79.

4. Liu L, Johnson HL, Cousens S, Perin J, Scott S, Lawn JE, Rudan I, Campbell H, Cibulskis R, Li M, Mathers C, Black RE, Child Health Epidemiology Reference Group of WHO and Unicef. Global, regional, and national causes of child mortality: an updated systematic analysis for 2010 with time trends since 2000. Lancet. 2012;379:2151-61.

5. Mekonnen Y, Tensou B, Telake DS, Degefie T, Bekele A. Neonatal mortality in Ethiopia: trends and determinants. BMC Public Health. 2013;13:483.

6. Teng JJ, Zhang J, Zhang TY, Zhang S, Li BS. Prognostic value of peripheral blood lymphocyte-tomonocyte ratio in patients with solid tumors: a meta-analysis. Onco Targets Ther. 2016;9:37-47.

7. Chiriac S, Stanciu C, Singeap AM, Sfarti CV, Cuciureanu T, Trifan A. Prognostic value of neutrophil-tolymphocyte ratio in cirrhotic patients with acute-on-chronic liver failure. Turk J Gastroenterol. 2020;31:868-76.

8. Smith RA, Bosonnet L, Raraty M, Sutton R, Neoptolemos JP, Campbell F, Ghaneh P. Preoperative platelet-lymphocyte ratio is an independent significant prognostic marker in resected pancreatic ductal adenocarcinoma. Am J Surg. 2009;197:466-72.

9. Wang C, He W, Yuan Y, Zhang Y, Li K, Zou R, Liao Y, Liu W, Yang Z, Zuo D, Qiu J, Zheng Y, Li B, Yuan Y. Comparison of the prognostic value of inflammation-based scores in early recurrent hepatocellular carcinoma after hepatectomy. Liver Int. 2020;40:229-39.

10. Proctor MJ, Morrison DS, Talwar D, Balmer SM, Fletcher CD, O'Reilly DS, Foulis AK, Horgan PG, McMillan DC. A comparison of inflammation-based prognostic scores in patients with cancer. A Glasgow Inflammation Outcome Study. Eur J Cancer. 2011;47:2633-41.

11. Liu Y, Zheng J, Zhang D, Jing L. Neutrophil-lymphocyte ratio and plasma lactate predict 28-day mortality in patients with sepsis. J Clin Lab Anal. 2019;33:e22942.

12. Karagoz I, Yoldas H. Platelet to lymphocyte and neutrophil to lymphocyte ratios as strong predictors of mortality in intensive care population. Rev Assoc Med Bras (1992). 2019;65:633-636.

13. Junare PR, Debnath P, Nair S, Chandnani S, Udgirkar S, Thange R, Jain S, Deshmukh R, Debnath P, Rathi P, Contractor Q, Deshpande A. Complete hemogram: simple and cost-effective in staging and predicting outcome in acute pancreatitis. Wien Klin Wochenschr. 2021.

14. Johnson AE, Pollard TJ, Shen L, Lehman LW, Feng M, Ghassemi M, Moody B, Szolovits P, Celi LA, Mark RG. MIMIC-III, a freely accessible critical care database. Sci Data. 2016;3:160035. 
15. Liu C, Zhou Y, He X, Ma J, Guo W, Dong B, Liang W, Wu Y, Owusu-Agyeman M, Xue R, Zhao J, Wu Z, Dong Y. Mean platelet volume/platelet count ratio predicts long-term mortality in patients with infective endocarditis. Biomark Med. 2020;14:293-302.

16. Nording HM, Seizer P, Langer HF. Platelets in inflammation and atherogenesis. Front Immunol. 2015;6:98.

17. Kim CH, Kim SJ, Lee MJ, Kwon YE, Kim YL, Park KS, Ryu HJ, Park JT, Han SH, Yoo TH, Kang SW, Oh $\mathrm{HJ}$. An increase in mean platelet volume from baseline is associated with mortality in patients with severe sepsis or septic shock. PLoS One. 2015;10:e0119437.

18. Manzoli TF, Delgado AF, Troster EJ, de Carvalho WB, Antunes AC, Marques DM, Zamberlan P. Lymphocyte count as a sign of immunoparalysis and its correlation with nutritional status in pediatric intensive care patients with sepsis: A pilot study. Clinics. 2016;71:644-9.

19. Felmet KA, Hall MW, Clark RS, Jaffe R, Carcillo JA. Prolonged lymphopenia, lymphoid depletion, and hypoprolactinemia in children with nosocomial sepsis and multiple organ failure. J Immunol. 2005;174:3765-72.

20. Akboga MK, Canpolat U, Yayla C, Ozcan F, Ozeke O, Topaloglu S, Aras D. Association of Platelet to Lymphocyte Ratio With Inflammation and Severity of Coronary Atherosclerosis in Patients With Stable Coronary Artery Disease. Angiology. 2016;67:89-95.

21. Zheng CF, Liu WY, Zeng FF, Zheng MH, Shi HY, Zhou Y, Pan JY. Prognostic value of platelet-tolymphocyte ratios among critically ill patients with acute kidney injury. Crit Care. 2017;21:238.

22. Akbas EM, Hamur H, Demirtas L, Bakirci EM, Ozcicek A, Ozcicek F, Kuyrukluyildiz U, Turkmen K. Predictors of epicardial adipose tissue in patients with type 2 diabetes mellitus. Diabetol Metab Syndr. 2014;6:55.

23. Wang YQ, Zhi QJ, Wang XY, Yue DS, Li K, Jiang RC. Prognostic value of combined platelet, fibrinogen, neutrophil to lymphocyte ratio and platelet to lymphocyte ratio in patients with lung adenosquamous cancer. Oncol Lett. 2017;14:4331-8.

24. Can E, Can C. The value of neutrophil-to-lymphocyte ratio (NLR) and platelet-to-lymphocyte ratio (PLR) parameters in analysis with fetal malnutrition neonates. J Perinat Med. 2019;47:775-9.

25. Akgun N, Namli Kalem M, Yuce E, Kalem Z, Aktas H. Correlations of maternal neutrophil to lymphocyte ratio (NLR) and platelet to lymphocyte ratio (PLR) with birth weight. J Matern Fetal Neonatal Med. 2017;30:2086-91.

\section{Figures}




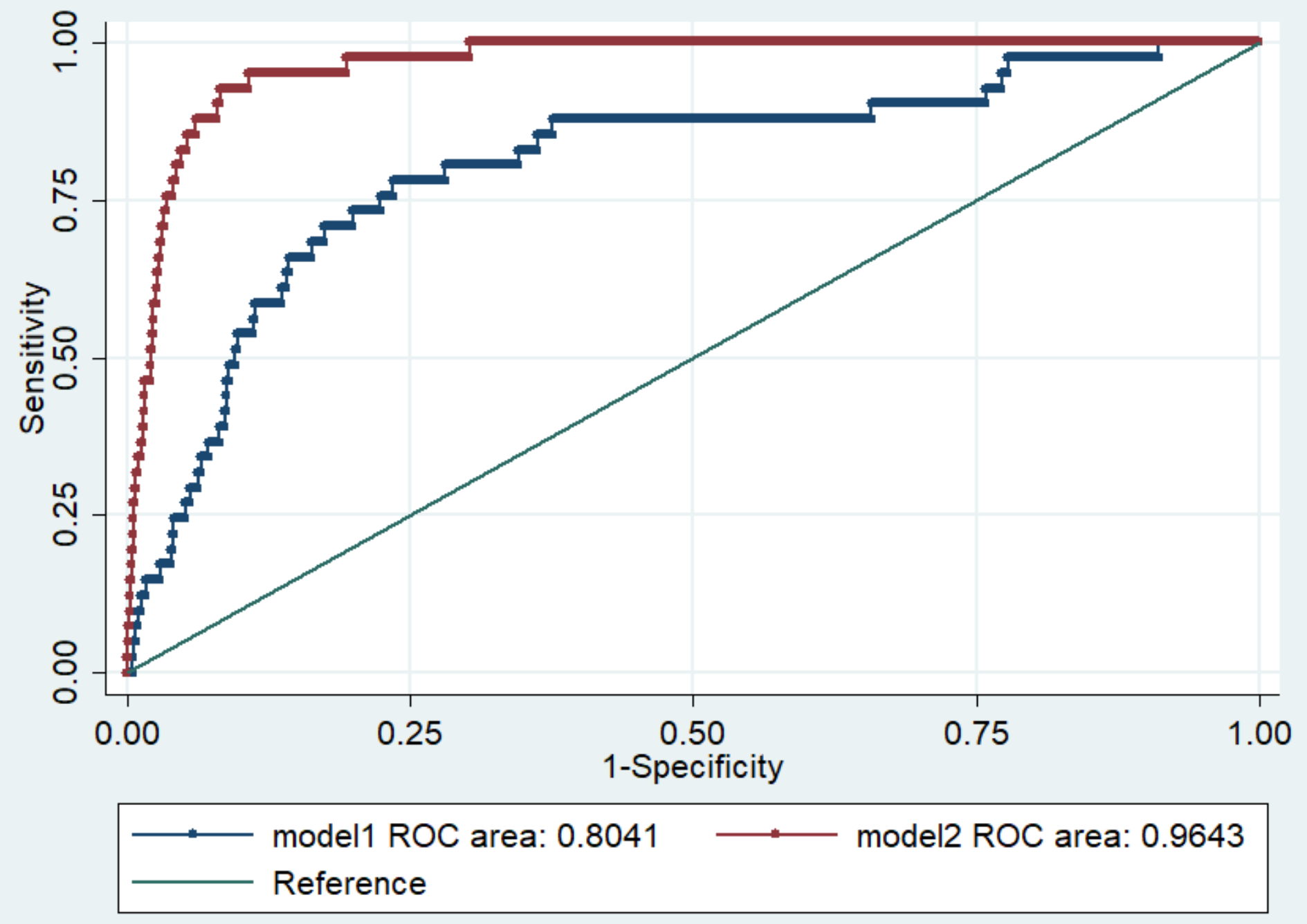

Figure 1

ROC curve for the predictive models for hospital mortality. Note: Model 1 included PLR, LMR and NLR. Model 2 included PLR, LMR, NLR, SOFA and MELD scores. AUC, area under the curve; ROC, receiving operating characteristic. 